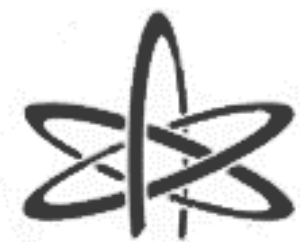

BJRS

\author{
BRAZILIAN JOURNAL \\ $\mathrm{OF}$ \\ RADIATION SCIENCES \\ 06-03 (2018) 01-15
}

\title{
Performance of detectors used in measurements of radioactive material deposited in tissues from intakes
}

\author{
by wounds
}

\author{
Lopes $^{\mathrm{a}}$ A.G., Bertellib L., Tauhata ${ }^{\mathrm{a}}$ L. \\ anstitute of Radiation Protection and Dosimetry (IRD)/National Laboratory of Ionizing Radiation Metrology (LNMRI), \\ CEP:22783-127, Rio de Janeiro, RJ, Brazil \\ ${ }^{b}$ Los Alamos National Laboratory (LANL)/Health Physics Measurements, Radiation Protection, NM 87545, \\ Los Alamos, USA \\ e-mail: amandglird@gmail.com
}

\begin{abstract}
In the care of people injured with wounds with incorporated radioactive material the basic information is collected, using appropriate detectors, following procedures established in first aid protocol. The reliability of the data collected for medical treatment depends greatly on the metrological performance of the equipment, the experimental setup and the scenario of the occurrence of the accident. The methodology to evaluate the performance of the detectors use the comparison of the attenuation coefficient value of each radionuclide obtained by the attenuation curve of tissue-equivalent material to the theoretical value established by the National Institute of Standards and Technology (NIST) in each experimental setup. The experimental setups were chosen according to the types of possible measurements performed in the various scenarios of the radiological accident. The detectors used were Personal Radiation Dosimeter, IdentiFINDER2, Cadmium Telluride and Hyper Pure Germanium. The results for ${ }^{241} \mathrm{Am}$ showed better performance with HPGe and IdentiFINDER2 shielded. For the ${ }^{60} \mathrm{Co}$ the best performance occurred with the shielded and collimated IdentiFINDER2. For the ${ }^{137} \mathrm{Cs}$, the HPGe detector presented the best performance.

Keywords: wounds contaminated, incorporation of radionuclides, performance of detectors, emergency radiological.
\end{abstract}




\section{INTRODUCTION}

In many research labs and facilities operating with radioactive materials people may be subject to accidents involving external and internal exposures, and sometimes through the incorporation of material through wounds. Events involving explosions of radioactive materials can cause such injuries, affecting operators, individuals and the environment. Such events can occur in a variety of settings, such as in specialized laboratories, or even in events involving members of the public due to the detonation of "dirty bombs".

According to the 2006 National Council on Radiation Protection and Measurements - NCRP [1], the scientific literature reports more than 2100 cases of wounds involving radionuclide incorporations, which occurred mostly in facilities involved in the production, manufacture or maintenance of components of nuclear weapons. The contaminants involved, mainly actinides (americium, plutonium and uranium). In more than $90 \%$ of the cases, they occurred in the regions of the hands and arms, but mainly in the fingers.

In the case of intake of radioactive material by wounds first aid must comply with established protocols involving measurements with detectors to identify and quantify the radionuclides, depth of incorporation and report, if possible, some physical-chemical properties such as composition, solubility and toxicity of the material. These data are important for further medical treatment. It is common to observe in these first aid calls that the measurements with the portable detectors lack accuracy and precision by the lack of appropriate shielding and collimation. A typical example occurred in February 1964 when a 26 year-old plutonium processing operator had a puncture of approximately 1 centimeter $(\mathrm{cm})$ into the palm of his right hand. After excision, residual wound activity was estimated at 1600 Becquerel (Bq). The last count in 2003, when the operator was 65 years old, the estimated activity was $3220 \pm 600 \mathrm{~Bq}$, value significantly higher than the initial activity, evidencing the difference in the quality of the detectors and procedures used [2].

This work aims to evaluate the performance of the detectors in the measurement of injuries with incorporated radioactive material, highlighting its advantages and mainly its limitations. 


\section{MATERIALS AND METHODS}

The evaluation of the performance of the detectors was made with measurements of the radiation energies emitted by radionuclides for several attenuations using different thicknesses of the tissue-equivalent material, representative of the possible scenarios of accident. The results were compared against the theoretical values established by the National Institute of Standards and Technology (NIST) of the United States of America.

The measurements were carried out in the Laboratory of Production of Radioactive Sources of the National Laboratory of Ionizing Radiation Metrology (LNMRI) at the Institute of Radiation Protection and Dosimetry (IRD).

The experimental data were obtained by simulating a radioactive fragment embedded in different depths of the body, using radioactive sources inserted in a tissue-equivalent material measured with different detectors and experimental arrangements [3].

\subsection{Choice of tissue-equivalent material}

The material chosen as the tissue-equivalent was polymethyl methacrylate (PMMA), better known as acrylic, which compared to other tissue-equivalent materials, possesses properties of scattering and absorption are very similar however, the attenuation of other materials are less than PMMA for energies of $60 \mathrm{keV}$ to $200 \mathrm{keV}$. The choice was based on the International Commission on Radiation Units and Measurements (ICRU) Publication 44 [4] and the NIST website. Ten plates of $0.2(\mathrm{~cm})$ each were used, totaling $2.0 \mathrm{~cm}$, which would simulate a situation of larger depth and allow the evaluation of the attenuation in the tissue with values obtained with 10 measurements for each thickness. 


\subsection{Accidents scenarios and radionuclides used}

The radionuclides and source formats chosen as representative of these scenarios. For this type of measurement, the attenuation associated to the thickness of the wall of the ampoule is not important because the data are express in A/Ao value. So, we measured only the exponential curve format. The characteristics of the standard sources used and possible scenarios are described in Tables 1 and 2.

Table 1: The characteristics of the standard sources used in the experiments.

\begin{tabular}{cccc}
\hline Radionuclides & Volume $(\mathrm{ml})$ & Activity $^{*}\left(\mathrm{kBq} \mathrm{g}^{-1}\right)$ & Shape/geometry \\
\hline${ }^{241} \mathrm{Am}$ & 2 & 9889.189 & ampoule \\
${ }^{60} \mathrm{Co}$ & 2 & 390.608 & ampoule \\
${ }^{137} \mathrm{Cs}$ & 2 & 18967.421 & ampoule \\
$\mathbf{U}_{\text {nat }}$ & $1\left(\mathrm{~cm}^{3}\right)$ & $999.85\left(\mathrm{mg} \mathrm{g}^{-1}\right)$ & solid \\
\hline
\end{tabular}

* Values at Reference Data

Table 2: Scenarios of possible accidents and representative radionuclides.

Scenarios of occurrence

Laboratories of production and handling of radionuclides

Nuclear artefacts and armament factories

Urban areas

\section{Representative radionuclides}

${ }^{137} \mathrm{Cs},{ }^{60} \mathrm{Co}$ and ${ }^{241} \mathrm{Am}$

${ }^{241} \mathrm{Am}$ and U-nat

${ }^{137} \mathrm{Cs}$ 


\subsection{Detectors used}

In this work were used two portable and two fixed detectors. The detectors were chosen because of their frequent use in field and emergency activities and in obtaining more accurate measurements in the monitoring of medical monitoring.

Portable detectors are used in field measurements because of their size and ease of locomotion and operation, for direct and immediate measurements. They are used for initial assessment of wound contamination and first aid measures.

Fixed detectors are used for monitoring and subsequent evaluation of biological samples, measured after initial decontamination and medical monitoring.

The detectors used were:

> Gamma Personal Radiation Detector (PRD) - Polimaster- PM 1703M;

$>$ IdentiFINDER2 - ICx Radiation Inc.- FLIR NGH;

> Cadmium Telluride (CdTe/ X) - AMPTEK Inc. - XR - 100T;

$>$ Hyper Pure Germanium (HPGe) - Canberra - GL2020R.

The Personal Radiation Detector (PRD) consists of a CsI (Tl) with volume of $3 \mathrm{~cm}^{3}$ coupled to a Geiger-Muller (GM). It provides measurements in counts per second (also represented as cps or count $\left.\mathrm{s}^{-1}\right)$ and in dose rate $\left(\mu S v \cdot \mathrm{h}^{-1}\right)$ values. It has high sensitivity for low energy values being able to detect a radioactive contamination, but does not identify the contaminating radionuclide.

The IdentiFINDER2 has a NaI (Tl) crystal size $35 \mathrm{~mm}(1.38 \mathrm{in}) \times 51 \mathrm{~mm}(2.01 \mathrm{in})$ coupled to a Geiger-Muller (GM) with ${ }^{3} \mathrm{H}$ tube. The material window is aluminum and it identifies the contaminating radionuclide and can display measurements in cps or in dose rate $\left(\mu \mathrm{Sv} \cdot \mathrm{h}^{-1}\right)$ values.

The Cadmium telluride (CdTe) is a diode-type detector used to detect photons between 30 and $200 \mathrm{keV}$, such as ${ }^{241} \mathrm{Am}$. The CdTe detector used was: Model XR-100- CdTe of the AMPTEK Inc. USA. The characteristics are: area: $5 \times 5 \mathrm{~mm}$, thickness: $2 \mathrm{~mm}$, window: Be $100 \mu \mathrm{m}$ and the dead layer: CdTe $0.15 \mu \mathrm{m}$. Its cooling is based on the Peltier effect [1] and generates radionuclide spectrum in cps per channel. 
The Hyper Pure Germanium detector (HPGe) used was the GL 2020 - Canberra with the following characteristics: area: $2000 \mathrm{~mm}^{2}$, thickness: $20 \mathrm{~mm}$, window: Be $0.5 \mathrm{~mm}$, and depleted depth: $16 \mathrm{~mm}$. The detector is allocated in a shield with the following materials and thicknesses: lead $(\mathrm{Pb}): 10 \mathrm{~cm}$, cadmium $(\mathrm{Cd}): 0.1 \mathrm{~cm}$ and copper $(\mathrm{Cu}): 0.08 \mathrm{~cm}$. It is cooled with liquid nitrogen.

The HPGe has an energy resolution of more than $400 \mathrm{eV}$ for X-rays of $5.9 \mathrm{keV}$ and $680 \mathrm{eV}$ for $\mathrm{X}$-rays of $122 \mathrm{keV}$, and allows the measurement of gamma radiations of up to $10 \mathrm{MeV}$. It is capable of identifying all radionuclides through of the gamma spectrometry system that consists of a GMX detector, preamplifier, amplifier, multichannel analyzer and the software Maestro for spectrum analysis. By the values of gamma energies and characteristic X-rays, the radioisotope is determined using the tables from the Laboratoire National Henri Becquerel (LNHB). Figure 1 illustrates the portable detectors used.

Figure 1: Portable detectors used: (a) PRD - Polimaster PM1703M and (b) IdentiFINDER2ICX Radiation Inc.- FLIR NGH.

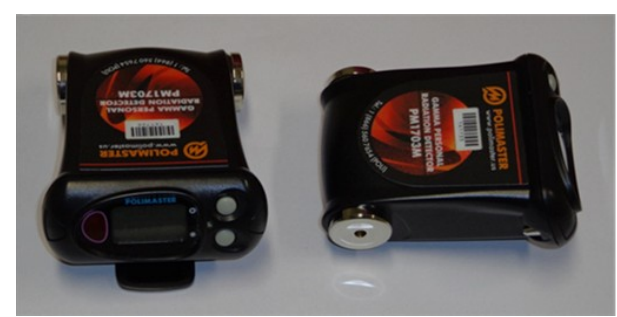

(a)

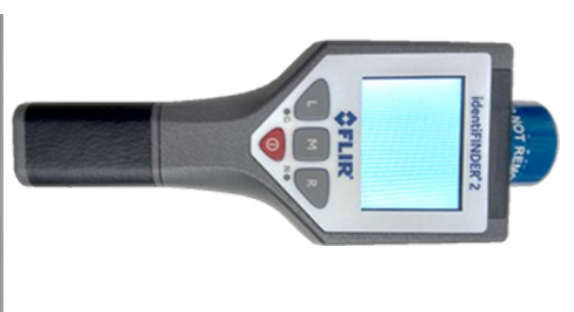

(b)

\subsection{Experimental setups}

In order to simulate the measurements performed in the various scenarios of radiological accidents three different experimental setups were used as shown in Figure 2, with appropriate use of collimator and shielding, in the measurements with the portable and fixed detectors. 
Figure 2: Experimental setups: (a) unshielded; (b) side shielding; (c) shielding + collimation.

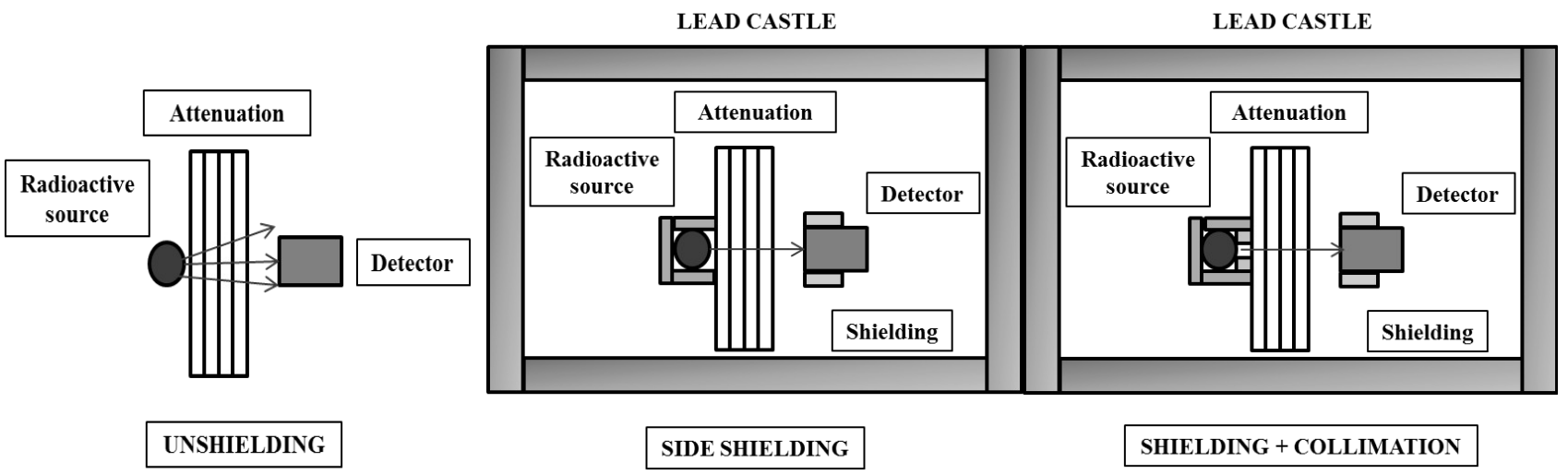

For all measurements the detector to source distance was $2 \mathrm{~cm}$ which was sufficient distant to allow adding 10 plates of $0.2 \mathrm{~cm}$ each. The used shielding was comprised of $\mathrm{Pb}, \mathrm{Cd}$ and $\mathrm{Cu}$ with their respective thicknesses of $10,0.1$ and $0.08 \mathrm{~cm}$.

\subsection{Measurements}

Estimating the quantity of activity embedded in wound requires identifying the radioisotope and the thickness of tissue between the source and detector. The quantity of activity directly measured using a detector near the wound must be adjusted to account for attenuation produced by overlying tissue. This adjustment is made by considering that attenuation of photons in tissue can be described using an exponential function with tissue thickness $(\mathrm{cm})$ and attenuation $\left(\mathrm{cm}^{-1}\right)$ as the exponent. Thus, the activity $\mathrm{A}_{0}$ of the fragment allocated to a depth $x$, is obtained from the activity $A$ measured at the wound site adjusted for attenuation using equation (1):

$$
A_{0}=A \cdot e^{\mu x}
$$


The value of the total attenuation coefficient $\mu(\mathrm{E})$ is a function of the effective energy of the radiation for the tissue-equivalent material and can be obtained using Table 4 of the Summary on the NIST website [6].

Thus, for each situation and radionuclide, attenuation curves which are associated with the depth or the thickness of the tissue can be obtained for each type of detector. Each type of detector produces its own attenuation curve which is different from another type due to measurement geometry, absence of shielding, collimation and its metrological properties.

The attenuation curves were constructed for each radionuclide and detector with 10 points, each point is obtained by the arithmetic mean of 10 measurements and their respective standard deviations. The graphs below show the measurements with their corresponding standard deviations. There are cases where the error bars are too small to appear in the graph. There is a note below each graph showing the maximum value for the uncertainty, in percentage, of each measurement.

The results of experimental coefficients were compared with the theoretical values provided by NIST.

\section{RESULTS AND DISCUSSION}

In order to do the interpretation of the measurements, the obtained values of the Total Attenuation Coefficient $(\mu)$ of the tissue-equivalent material with different detectors, the experimental setup and the radionuclides were used and compared against the theoretical values established by the NIST.

\subsection{Presentation of the results by measured radionuclide}

$$
{ }^{241} \mathrm{Am}
$$

Figures 3 and 4 show the attenuation curves obtained for the ${ }^{241} \mathrm{Am}$ measurements with different types detectors and different experimental setups. 
Figure 3: Results from the portable detectors for the ${ }^{241} \mathrm{Am} 60 \mathrm{keV}$ photon radiation.

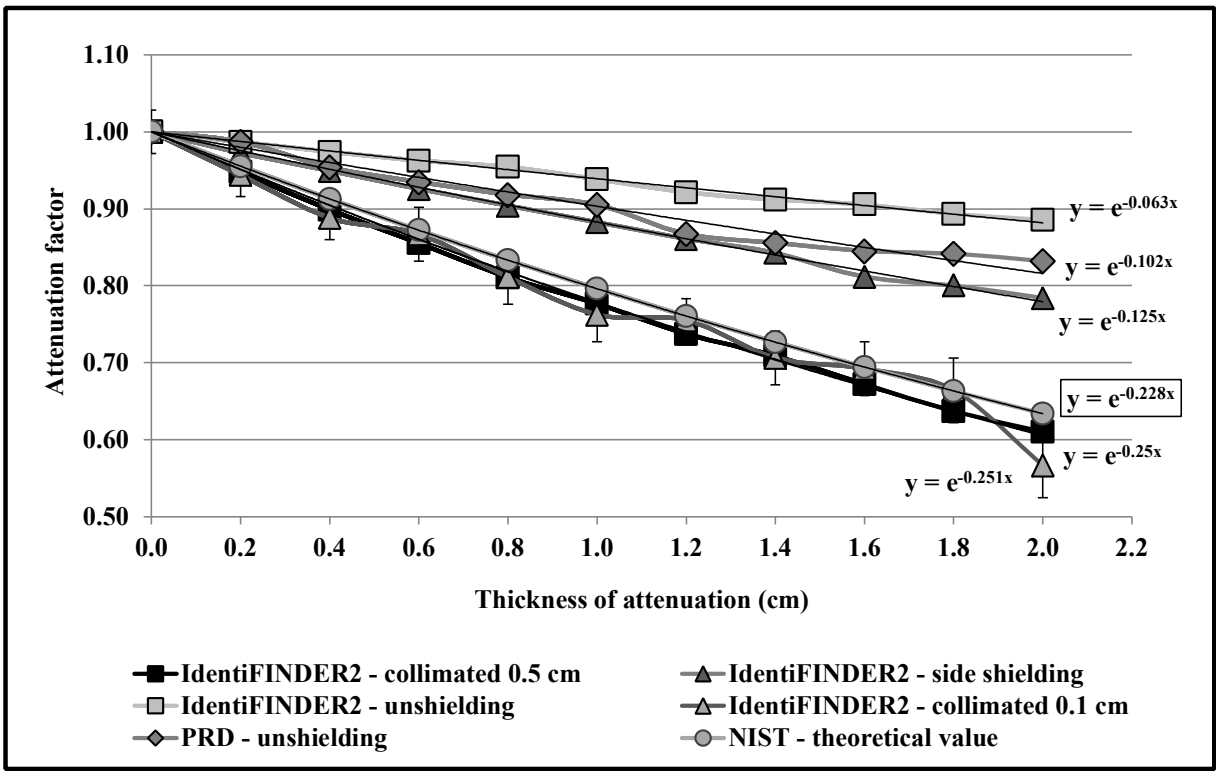

*The uncertainty values were expressed by the standard deviation and were less than $4.5 \%$.

Figure 4: Results from the fixed detectors for the ${ }^{241} \mathrm{Am} 60 \mathrm{keV}$ photon radiation.

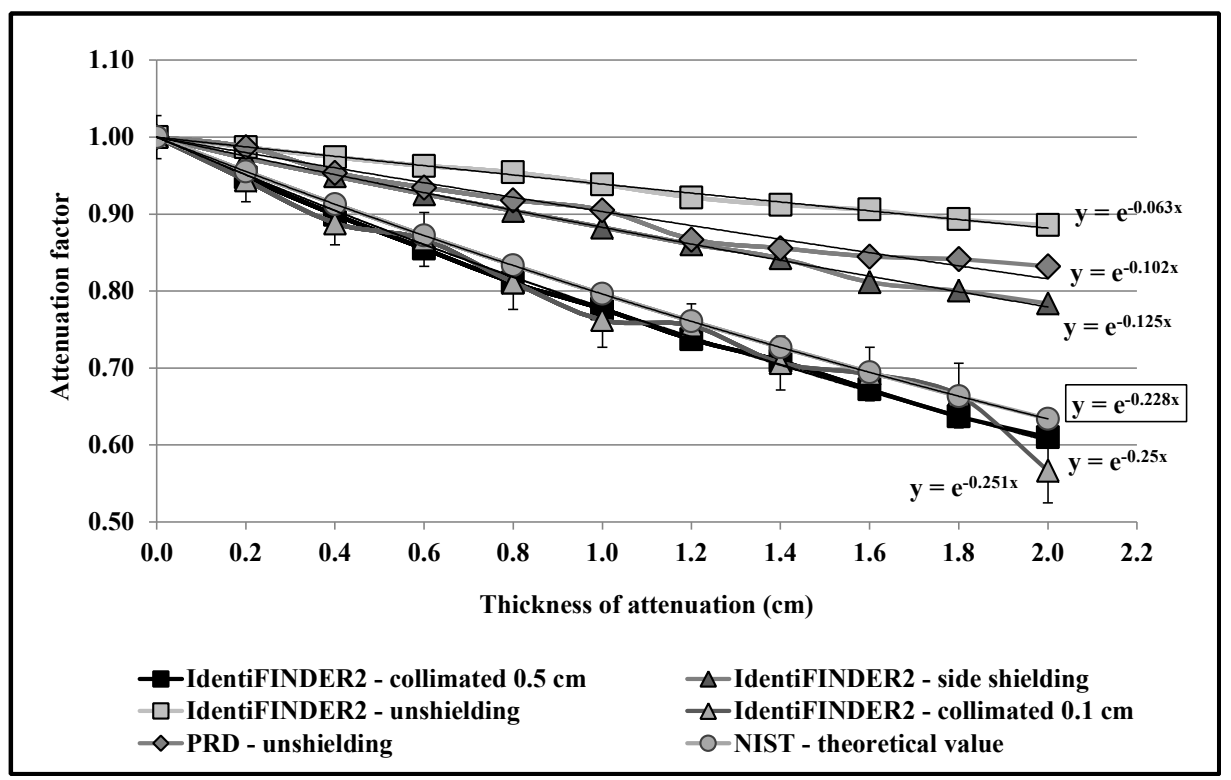


*The uncertainty values were expressed by the standard deviation and were less than $5.5 \%$.

The values of the attenuation coefficient obtained by the portable detectors are different from those presented by the NIST. However, when a collimation was added, this value approached the theoretical value.

The attenuation curves obtained with the fixed detectors HPGe and CdTe presented better values for the attenuation coefficient. Because the HPGe has a good resolution and accuracy the obtained value with shielding was $0.244 \mathrm{~cm}^{-1}$ that is $1.6 \%$ of the NIST value of $0.248 \mathrm{~cm}^{-1}$. The CdTe was not shielded and it has resulted in $0.163 \mathrm{~cm}^{-1}$, which is $1.9 \%$ smaller.

\section{${ }^{60} \mathrm{Co}$}

Figure 5 shows the attenuation curves obtained for the ${ }^{60} \mathrm{Co}$ measurements with the portable detectors having different experimental setups.

Figure 5: Results from different detectors for the ${ }^{60}$ Co radiation.

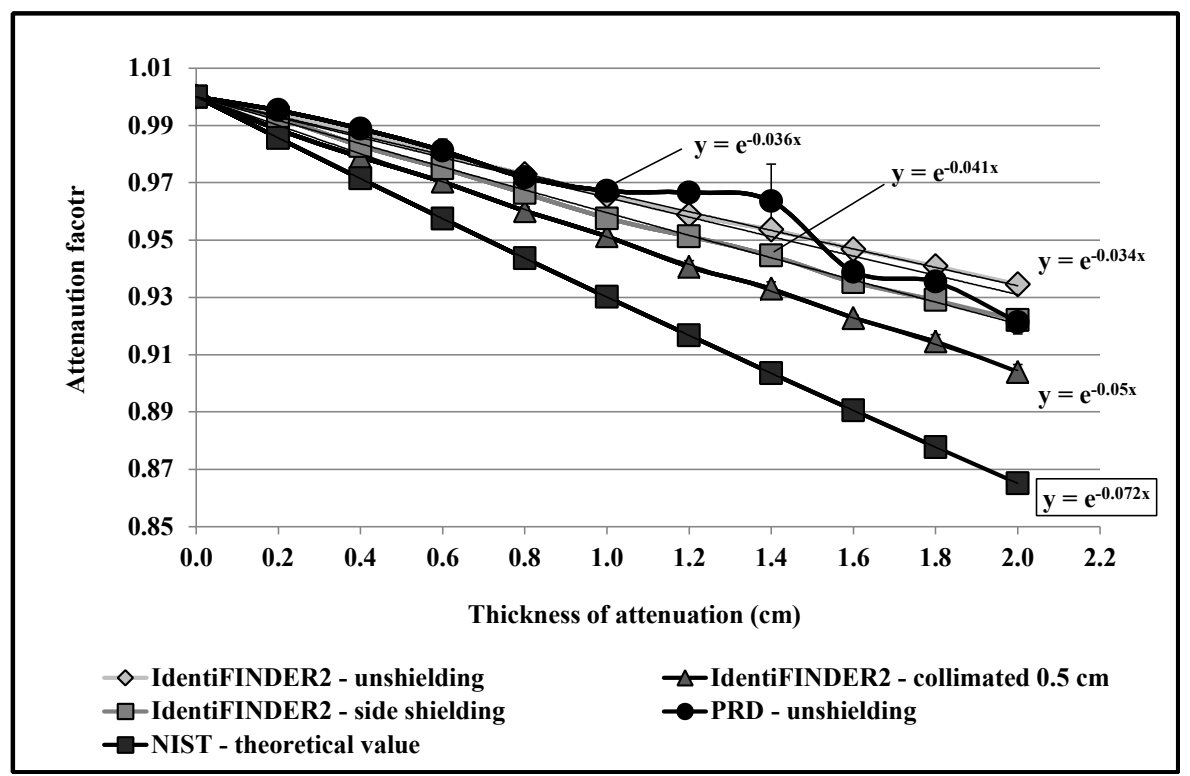

*The uncertainty values were expressed by the standard deviation and were less than $0.5 \%$. 
The values obtained with the two unshielded detectors are far from the theoretical value. So, for the NIST value of $0.076 \mathrm{~cm}^{-1}$, the IdentiFINDER2 presented the value $0.034 \mathrm{~cm}^{-1}$ and the PRD, $0.036 \mathrm{~cm}^{-1}$. When the collimation was introduced the result was $0.05 \mathrm{~cm}^{-1}$.

\section{${ }^{137} \mathrm{Cs}$}

Figure 6 shows the attenuation curves obtained with the measurements from the different detectors used for the ${ }^{137} \mathrm{Cs}$.

Figure 6: Results from the detectors for the $661 \mathrm{keV}^{137} \mathrm{Cs}$ photon.

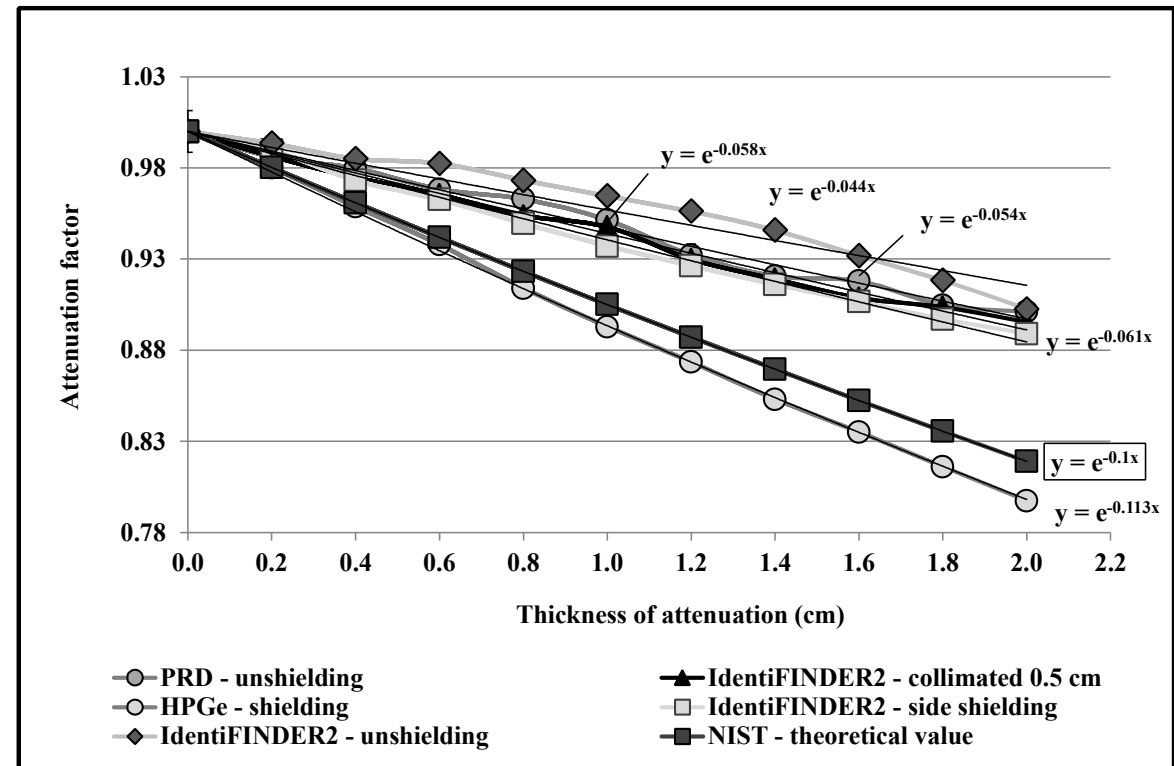

*The uncertainty values were expressed by the standard deviation and were less than $1.2 \%$. 
As can be seen the best result was obtained by measuring with HPGe and the worst was with the unshielded IdentiFINDER2 detector.

\section{Natural uranium}

Figures shows the attenuation curves obtained with energy of $184.98 \mathrm{keV}$ with the HPGe and the theoretical value. 
Figure 7: Results from the HPGe for energy of $184.98 \mathrm{keV}$ of the natural uranium.

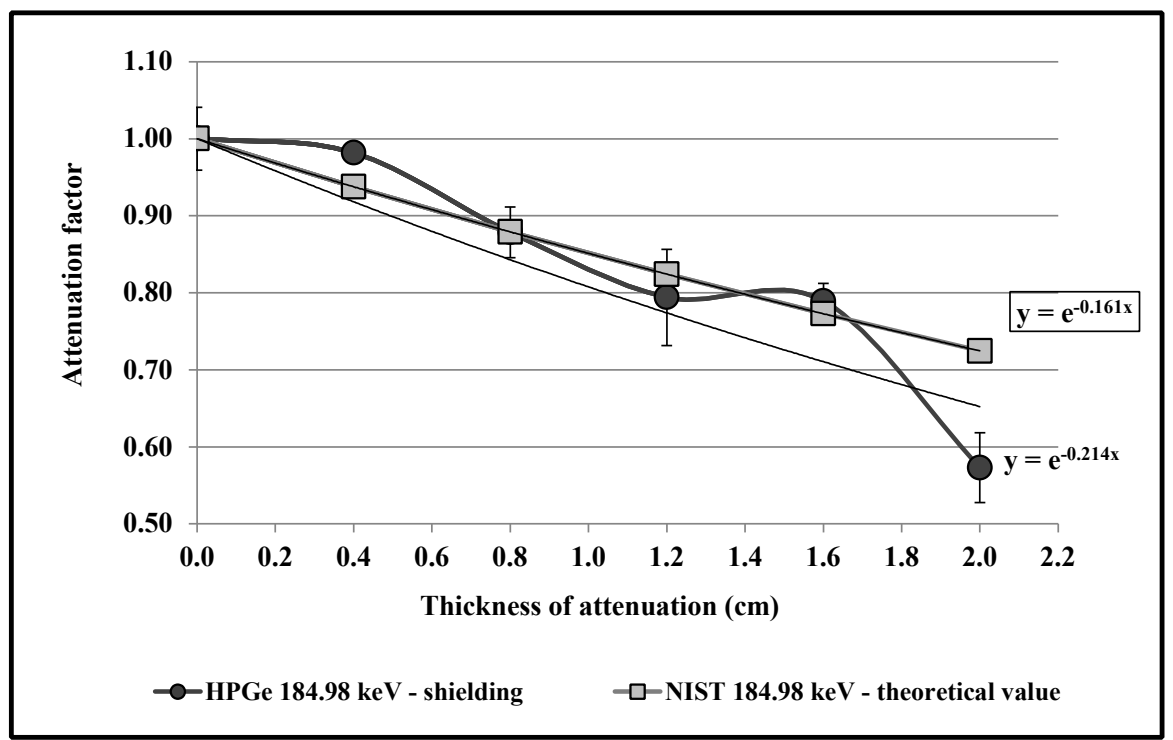

*The uncertainty values were expressed by the standard deviation and were less than $6.5 \%$.

Due to the shape and geometry of natural uranium that was used, the attenuation coefficient obtained was higher than the theoretical value established by NIST.

\subsection{Discussion of the results}

As expected, the results with portable and unshielded instruments, which represent the majority of first-aid measurements of accident victims, are those that presented the largest percentage deviations from the theoretical values.

Another important factor was the energy value of the photon radiation. Measurements of lowenergy radiation, whether characteristic X-rays or gamma radiation, are those that are most influenced by external factors such as background radiation and lack of collimation.

The values obtained for the total attenuation coefficient $(\mu)$ of the acrylic, when compared to the theoretical value established by the NIST, show that the more refined the experimental arrangement the more the results are close to the ideal ones. 
Table 3 shows the deviation between the experimental and the theoretical value as function of the absorber thickness.

Table 3: Percentage deviation of the experimental values in relation to the established by NIST as a function of absorber thickness $\mathrm{x}$ for each radionuclide and type of detector used. 


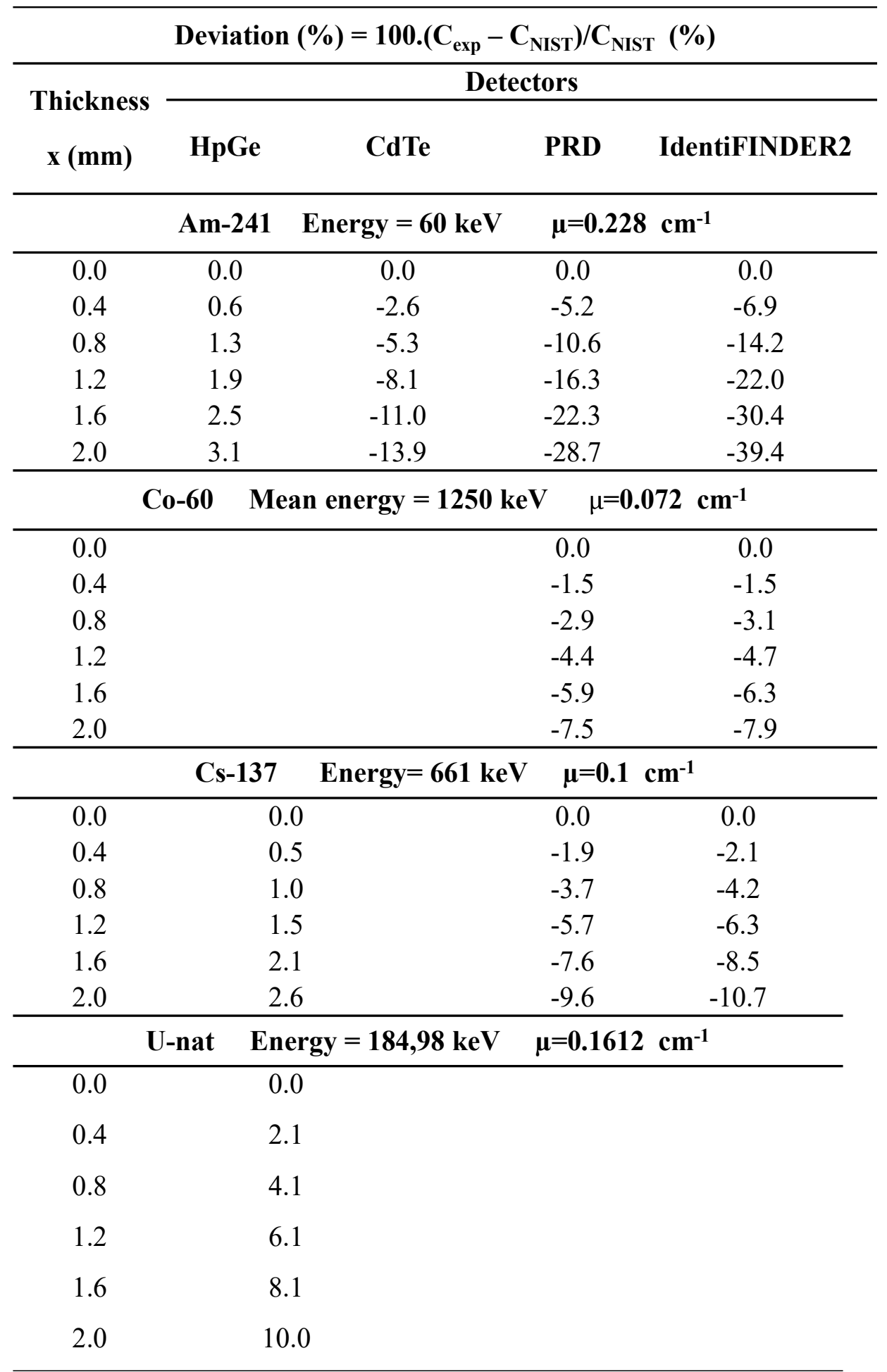


The values obtained show that the difference between the experimental and the NIST values increases with the thickness of the absorber. So, in the wound this difference will depend on the depth of the region that suffered the damage. Although this increases with all the detectors used, the fixed ones presented smaller values than the portable ones. For the ${ }^{241} \mathrm{Am}$, measured with four different detectors, simulating the worst case with a contamination at a depth of $2.0 \mathrm{~cm}$, the smallest difference was obtained by the HPGe and the biggest with the IdentiFINDER2. These results in a first-aid care are as useful as the high precision and accuracy measurements obtained by the fixed detectors since they serve to guide the decision-making associated with the care protocol.

\section{CONCLUSION}

This work made possible to evaluate the performance of detectors in situations of injuries contaminated by radionuclides through the analyses of their limitations and deficiencies. The use of shielding and collimation was efficient to approximate the experimental to the NIST value for the radionuclides used.

In these measurements, the main purpose is to show a difference of values obtained in measurement using portable detector in comparison with the laboratory measurement with more precise detector like GMX. The introduction of collimation is only to show that it is influence for to obtain for best results.

In general, the uncertainties presented values lower than $1.5 \%$ for a $68 \%$ confidence level $(\mathrm{k}=$ 1) under laboratory conditions. However, in field measurements it may present values up to 10 times higher due to the difficulties, lack of shielding and collimation and good geometry of measurement.

The choice for a portable or fixed detector will depend on the intended objectives. The portable detectors provide a qualitative response, whether or not it is contaminating, since the fixed detectors provide a quantitative response and with results with precision and accuracy.

However, although portable detectors do not provide precise and accurate measurements, they provide estimative dose values, which from the point of view of radiation protection is a conservative method and are useful for establishing future medical procedures for victims. 


\section{ACKNOWLEDGMENT}

The authors are grateful for the financial support from CNEN regarding the scholarship of the student Amanda Gomes Lopes, which made this work possible.

\section{REFERENCES}

[1] NCRP - National Council on Radiation Protection and Measurements. Development of a Biokinetic Model for Radionuclide-Contaminated Wounds and Procedures for their Assessment, Dosimetry and Treatment. NCRP Report 156, Bethesda: NCRP, 2006.

[2] FAlK, R. B.; DAUGHERTY, N. M.; ALDRICH, J. M.; FURMAN, F. J.; HILMAS, D. E. Application of Multi-Compartment Wound Models to Plutonium-Contaminated Wounds Incurred by Former Workers at Rocky Flats. Health Phys, v. 91(2), p. $128-143,2006$.

[3] LOPES, A. G.; TAUHATA, L.; BeRTElli, L., Protocolo para trato de pessoas que sofreram ferimentos com material radioativo em acidentes radiológicos e nucleares. Dissertação de Tese de Mestrado. Rio de Janeiro: IRD, 2015.

[4] ICRU - International Commission on Radiation Units and Measurements. Tissue substitutes in radiation dosimetry and measurements. ICRU Report 44, Bethesda: ICRU, 1989.

[5] LNHB - Laboratoire National Henri Becquerel. Recommended data. Available at: $<$ http://www.nucleide.org/DDEP_WG/DDEPdata.htm>. Last accessed: 15 December. 2015.

[6] NIST - National Institute of Standards and Technology. X-Ray Mass Attenuation Coefficients. Available at: $<$ http://physics.nist.gov/PhysRefData/XrayMassCoef/tab4.html $>$. Last accessed: 15 December. 2015. 